\title{
Mythiques chaînes opératoires
}

\section{Pierre Lemonnier}

\section{OpenEdition}

Journals

Édition électronique

URL : https://journals.openedition.org/tc/1054

DOI : $10.4000 /$ tc. 1054

ISSN : 1952-420X

\section{Éditeur}

Éditions de l'EHESS

\section{Édition imprimée}

Date de publication : 1 décembre 2004

ISSN : 0248-6016

\section{Référence électronique}

Pierre Lemonnier, « Mythiques chaînes opératoires », Techniques \& Culture [En ligne], 43-44 | 2004, mis en ligne le 15 avril 2007, consulté le 29 septembre 2022. URL : http://journals.openedition.org/tc/1054 ; DOI : https://doi.org/10.4000/tc.1054

Ce document a été généré automatiquement le 29 septembre 2022

Tous droits réservés 


\title{
Mythiques chaînes opératoires
}

\author{
Pierre Lemonnier
}

1 Qu'il s'agisse d'incunables (Cresswell 1972, Leroi-Gourhan 1964, Geistdoerfer 1973), de précis savants (Balfet 1975, 1991), de recueils de vœux pieux (Lemonnier 1976) ou de credo revivalistes (Digard 1979), les textes sont formels : la chaîne opératoire constitue la matière première de l'ethnologie des techniques. Pour qui prend au sérieux la proposition générale de Mauss (1968) d'élargir le champ de l'ethnologie à ces humbles et triviaux comportements humains que sont les techniques du corps, et, a fortiori à toute action technique, comprendre en quoi une opération matérielle est propre à un groupe particulier, c'est d'abord tenter de déchiffrer la manière dont divers éléments (énergies, outils, gestes, connaissances, acteurs, matériaux) sont mis en relation au cours de processus qui modifient un système matériel: disons la fabrication d'une poterie, la mise en vol d'un aéroplane ou la préparation du café du matin -pour reprendre un exemple «moderne » qui a épuisé la patience de cohortes d'étudiants. Pourquoi «d'abord»? Tout simplement parce que, à trop centrer l'étude sur les multiples manières dont les techniques font autre chose que modifier des corps, des objets ou notre environnement, on risque d'oublier en chemin une partie du programme de l'ethnologie, qui est de rendre compte de la diversité relative des productions socioculturelles des êtres humains, quelles qu'elles soient, ici et maintenant ou hier et là-bas, y compris celles qui fleurent bon le cambouis. Sont ainsi prises en compte par la technologie culturelle, la manière dont ces éléments interfèrent physiquement (c'est-à-dire tels que peuvent les décrire une physique et une chimie suffisamment ordinaires et indiscutables pour que chacun y entrevoie des vérités et des faits), mais aussi la façon dont les acteurs "pensent" de tels systèmes, singulièrement dans le but de les mettre en œuvre, mais pas seulement.

Désignant à la fois la succession de gestes et d'opérations matérielles effectuées par un acteur (ou par la machine qui prolonge sa pensée autant que celle de ceux qui l'ont conçue) et la représentation simplifiée que s'en fait un observateur, la notion de chaîne opératoire est trompeuse. De plus, sachant que la culture matérielle d'une société donnée forme un système au sein duquel de multiples formes d'actions sur la matière sont reliées les unes aux autres (Gille 1978, Lemonnier 1983), décider où commence et 
finit une technique est toujours un choix ad hoc du chercheur, qui définit le grossissement de son observation, le séquençage temporel et les bornes matérielles de l'action décrite, ce qui implique d'avoir un minimum de compréhension de phénomènes physiques et chimiques en jeu dans ce que l'on observe. La reconstitution d'une chaîne opératoire n'en est pas moins un moyen plutôt simple de caractériser les éléments et les étapes d'une transformation matérielle contrôlée par un agent (humain ou primate, d'ailleurs). À l'échelle des phénomènes dont ils ont à rendre compte, les ethnologues et les archéologues ne font pas autre chose qu'appliquer des méthodes semblables à celle des ingénieurs des bureaux d'études et des spécialistes de recherche opérationnelle qui prévoient les étapes de processus de façonnage ou de fabrication, dont l'ampleur temporelle et la complexité spatiale dépassent de plusieurs ordres de grandeur celle des techniques que les chercheurs en sciences humaines ont habituellement à connaître.

Nul besoin d'être grand sage, cependant, pour noter que la description et l'analyse des chaînes opératoires ne font plus guère recette. Ces proches cousins de l'ethnologie des techniques que sont les sociologues de l'innovation n'ont guère valorisé la description pas à pas de la production ou de l'emploi des objets dont ils étudient (à juste titre) l'imbrication dans toutes sortes de modes de constitution du lien social. De même, et bien que la "culture» dont ils tentent de saisir les linéaments soit largement constituée de techniques du corps et de la mise en œuvre d'un tool kit plus que basic, les primatologues ne se montrent pas plus empressés à décrire par le menu les faits et gestes des animaux qu'ils observent ${ }^{1}$. Joulian (1996) ou Byrne (1995) font évidemment exception, mais force est de constater que la méthode appliquée dans les célèbres articles comparatifs de Nature et Behaviour (Whiten et al. 2000, 2001) consacrés aux "cultures" matérielles des chimpanzés, relève bien davantage d'une approche en termes de «traits caractéristiques » et de diffusion de ceux-ci, qui n'aurait pas déparé dans les années 1930, que d'une lecture assidue de Techniques \& culture (Lemonnier 2004). En ethnologie même, les processus techniques n'ont pas retenu l'attention des anthropologues marxistes de naguère qui, plus que d'autres, auraient dû se pencher sur la dimension la plus matérielle des forces productives en même temps qu'ils analysaient les rapports sociaux de production ${ }^{2}$. D'une manière plus inquiétante -ou qui ne peut manquer de faire réfléchir-, les meilleures des material culture studies font également l'impasse sur la description et l'analyse de la production des objets dont les formes de " consommation » sont examinées (par exemple Faure-Rouesnel 2001, Julien et al. 2002, Küchler 1997, Warnier 1999). Un récent numéro de L'Homme démontre même qu'il est désormais parfaitement légitime de développer une anthropologie des objets dont ni la fabrication ni les usages premiers ne sont abordés, mais seulement leur devenir une fois récupérés par des collectionneurs, investis de fonctions non prévues par leurs fabricants ou dotés par leur itinéraire propre d'un statut de " presque-plusrien " (selon l'expression de Debary, Tellier 2004). Quant à la revue Techniques \& culture, héraut s'il en fut de l'analyse des chaînes opératoires, elle ne publie plus que de loin en loin des recherches fondées sur l'analyse de processus matériels décrits par le menu. Bref, à de remarquables exceptions près (Gosselain 1999, Küchler 1988, Leach 2002), la majeure partie des travaux d'ethnologie ou de sociologie abordant de près ou de loin la vie matérielle fait plus que jamais l'impasse sur la dimension la plus matérielle des comportements techniques. Seuls les archéologues font un usage constant des problématiques et instruments développés par Balfet et Cresswell dans la ligne de Leroi-Gourhan. 
4 Sauf erreur, ni la sociologie de l'innovation ni les material culture studies n'ont jamais proposé de critique construite de l'analyse des chaînes opératoires, mais leur lourd silence à propos de celles-ci revient à clamer l'inutilité d'une approche partiellement fondée sur leur étude. L'ethnologie n'a jamais rejeté cette approche, mais tout patient observateur de potières, d'archers ou de cuisiniers sait d'expérience qu'il ou elle n'utilisera jamais qu'une infime partie des chaînes opératoires engrangées au péril de l'ennui sous forme de notes, croquis, photographies, films et enregistrements sonores. Si ces inutiles et fastidieux concentrés de terrain ne sont que des bribes d'une ethnographie de longue durée, la chose importe peu. Mais que dire des documents inutilisés par les étudiants que nous avons sévèrement enjoints de braver la pluie, le soleil et la fatigue pour comprendre et traduire de leur mieux les gestes techniques de leurs hôtes? Trésors ou improbables archives?

Ce décalage entre la place que les créateurs de la présente revue revendiquaient naguère pour l'analyse des chaînes opératoires et la pratique contemporaine de celles des sciences humaines qui se soucient de la dimension culturelle des actions sur le monde matériel conduit à se demander si les chaînes opératoires ne seraient pas des mythes. Leur "recueil" ne serait-il qu'un acte rituel qu'une poignée de fanatiques tentent d'imposer à des cadets? Bref, les chaînes opératoires sont-elles des logarithmes jaunes? Ou, au contraire, de profitables instruments de description et d'analyse dont l'ethnologie aurait tort de se passer, y compris lorsqu'elle se tourne vers des objets high-tech tels que les sites Internet ou les soins aux prématurés ${ }^{3}$ ?

Mon propos est évidemment d'illustrer cette dernière proposition, mais en me plaçant pour une fois -et par un souci de symétrie quasi latourien - sur le terrain de ceux pour qui la dimension matérielle des techniques serait un trivial à-côté de la production des systèmes de sens et de pratiques : en prenant pour point de départ deux ensembles de mythes et de rites des Ankave-Anga dont l'analyse et la compréhension resteraient obstinément obscures sans une fine connaissance des chaînes opératoires permettant de produire deux objets techniques des plus ordinaires en Papouasie Nouvelle-Guinée.

Des tambours qui ne font pas que du bruit

7 Cinquante ans après avoir découvert l'existence des Blancs et malgré la présence épisodique de ces catéchistes papous qu'ils finissent toujours par mettre à la porte de leurs vallées, les Ankave continuent de penser ensemble ces phénomènes cruciaux que sont l'imputation du malheur, les représentations de la maladie, les attaques cannibales imaginaires, le travail des chamans et le respect des bonnes manières. La gestion du malheur, les logiques de l'échange et les procédures de deuil se trouvent régulièrement associées lors de cérémonies qui permettent à ce petit peuple d'horticulteurs forestiers de chasser à jamais, croit-on, les spectres errants des morts récents (pisingen siwi).

8 Ces cérémonies, qui portent le nom des tambours qu'on y fait résonner nuit après nuit (songain), sont la partie la plus ostensible d'un système de pensée au cœur duquel se trouvent des êtres immondes, cannibales, invisibles et foncièrement méchants, les ombo', que les Ankave tiennent pour responsables de la majorité des maladies mortelles. Ontologiquement, ce ne sont ni des humains ni des esprits, mais l'association sui generis d'un humain (homme, femme ou enfant ayant atteint l'âge de raison) et d'un esprit cannibale. Ils constituent une affreuse bande d'affamés anonymes qui jouent deux rôles sociologiques essentiels. D'abord, parce qu'ils attaquent, dévorent et se partagent des hommes, des femmes et des enfants dont on pense qu'ils avaient eux-mêmes été de mauvais partageurs, les ombo' sont un constant rappel de ce pilier de l'ordre social 
ankave qu'est l'obligation de répondre favorablement à toute requête concernant de la nourriture ou un bien. Ensuite, ils sont ceux qui se chargent de la disparition des chairs des cadavres, qu'ils sont supposés dévorer.

Mais les mythes rapportent également que c'est aux ombo' que les humains doivent l'instauration des cérémonies songain. Ce sont eux qui, du fond d'une mare, ont procuré à l'humanité les tambours sabliers que l'on doit battre toutes les nuits lorsqu'est venu le temps de faire fuir à jamais un esprit pisingen siwi, et d'oublier le défunt dont il est le spectre; on leur doit aussi les masques de ceux qui jouent les tambours, ainsi que les chants que l'on entonne en battant ceux-ci. En même temps que les songain, volés dit-on aux ombo', les hommes ont découvert la vie brève, la sexualité, les dons qui accompagnent le mariage... L'affaire, on le voit, est d'importance. Ce sont ces mêmes tambours que les Ankave utilisent aujourd'hui, réparant ou fabriquant de nouveaux instruments chaque fois que cela est nécessaire, c'est-à-dire une ou deux fois par an dans chacune des trois vallées qu'ils occupent.

10 Un tambour ankave (Papouasie Nouvelle-Guinée) s'apparente à un long sablier (77 à $120 \mathrm{~cm}$ pour les exemplaires que j'ai vus) constitué de deux cônes se rejoignant par leurs sommets et surmontés d'une poignée. Sa partie supérieure, sur laquelle on frappe, présente un diamètre maximum de 11 à 13 centimètres tandis que celui de l'ouverture par laquelle s'échappe le son atteint 12 à 16 centimètres. La membrane du tambour et les petites boules de cire que l'on y colle pour l'accorder reçoivent le nom des matériaux dont ils sont constitués (sindre' waa', " peau de serpent" et unda'a, "cire d'insecte "), mais d'autres parties de l'instrument sont décrites à l'aide du vocabulaire appliqué aux corps, humains ou animaux: "épaule" (saongwain) pour la poignée ; "bouche" (mangain) pour l'ouverture sur laquelle est collée et ficelée la membrane; "cul" (ike' mangain) pour l'ouverture. L'étranglement central du tambour est son " cou " (ngu'no'). Les cônes eux-mêmes sont l'un et l'autre décrits comme des " milieux" ( $a^{\prime}$ wone'), au sens de "parties intermédiaires". De prime abord, un tambour ankave est un instrument de musique. À y regarder de plus près -c'est-à-dire en menant une enquête de technologie culturelle pendant qu'il en est encore temps-, les choses sont plus compliquées.

11 Pour l'ethnologue spécialisé dans l'étude des rapports entre techniques, culture et société, la préparation de ce temps fort de la vie ankave que sont les rites songain est l'occasion de découvrir l'une de ces chaînes opératoires qui constituent le point de départ habituel des recherches de technologie culturelle. Avec une particularité de taille cependant : la chaîne opératoire en question n'est pas de celles que l'observateur regarde, décrit et transcrit dans ses carnets de notes. Elle est donnée par les Ankave eux-mêmes, sous la forme d'une série de mythes qui expliquent, étape par étape, comment fabriquer l'instrument, en même temps qu'ils soulignent des aspects-clefs du dispositif imaginaire par lequel cette population évacue ses morts: l'origine de la membrane sonore, faite d'une peau d'homme-serpent, et l'importance du "cou ", ou partie centrale de l'objet (Lemonnier s/presse).

12 Après avoir indiqué qu'un lointain ancêtre se rendit auprès d'une mare tourbillonnante d'où sortait un son aussi étrange que merveilleusement beau, le mythe raconte comment, n'ayant pu saisir un premier tambour surgi de l'eau, le héros s'empare d'un second instrument :

En même temps que ce tambour, la mare lui a donné, ficelé sur l'extérieur de

l'objet : une feuille de l'arbre wuyae'wa [Gmelina sp.], dans le bois duquel on fait les 
tambours; une liane nongwaye qui a des épines [un rotin, Calamus sp.]; une feuille de l'arbre so'o [Pipturus argenteus], dont on tire de la colle; un couteau de bambou, pour gratter le bois du tambour; et un bâtonnet en bois de miye', un arbre dur [Garcinia sp.], pour percer le tambour; une peau de serpent "en place" [sans doute tendue sur un séchoir]; et l'insecte qui donne la cire unda'a'. Tout cela était ficelé sur le tambour.

L'homme a regardé toutes ces choses que l'eau elle-même avait accrochées au tambour. Il les a prises et les a " essayées " [c'est-à-dire qu'il a cherché à quoi elles servaient] en se cachant. Il a dissimulé l'instrument sous sa cape d'écorce bien serrée contre lui. Et il l'a camouflé dans sa maison. frappant des entrenœuds de bambou sur le sol.

Les autres étaient en train de cuire leur dîner sur les lieux de la cérémonie. Il a pris le tambour qu'il avait caché et un peu de nourriture et il est allé les rejoindre. Les autres se sont dit qu'il apportait à manger. Il est arrivé avec le tambour. Il a retiré le morceau d'écorce battue qui le recouvrait et a frappé l'instrument. Tous ceux qui cognaient les bambous sur le sol l'ont regardé : c'était un son différent. Ils étaient complètement abasourdis! Et l'homme chantait en même temps. Il chantait: "Nerwambonaa rwambewayo nerobewa topewayo" [c'est-à-dire le refrain du tourbillon]. Chacun a alors jeté son tube de bambou. Lui seul a battu tambour et chanté toute la nuit devant les autres, qui le regardaient. L'aube vint, tout le monde se rassembla et observa le tambour et ce que l'eau y avait attaché.

Ils ont compris en regardant et retirant tout ça [ce qui était attaché], et en voyant le tambour [lui-même]. Ils ont respiré l'odeur des feuilles et su quel était l'arbre. Tous sont partis à la recherche de cet arbre avec l'homme au tambour. Ils en coupaient un et le sentaient, mais ce n'était pas le bon! Puis ils ont trouvé le wuyae'wa et l'ont senti : cet arbre avait la même odeur que les feuilles attachées au tambour. Ils ont comparé les feuilles : c'étaient les mêmes. Ils ont abattu l'arbre, qui est tombé. Chacun en a pris un morceau après avoir posé le tambour sur le tronc pour connaître la bonne longueur à couper [pour chaque segment]. Ils ont entièrement débité l'arbre. Ils ont voulu mettre ces bouts de bois en forme, alors ils ont regardé ce que l'eau leur avait révélé. Ils ont repéré l'arbre miye', l'ont coupé et ont percé les tambours, chacun le sien [le miye' possède un bois avec lequel on creuse progressivement les cavités de l'instrument après en avoir brûlé l'intérieur]. Puis ils ont retourné le tambour pour en percer l'autre extrémité, puis la partie centrale, avec le miye'. Ils ont ressorti le miye' et pris un morceau du rotin à épines, avec lequel ils ont limé l'intérieur de la pièce de bois, jusqu'à faire un grand trou. Puis ils ont allumé du feu dans cette ouverture, tout en installant un bouchon de terre pour en contrôler la combustion. Alors, ils ont soufflé sur les braises. Quand le trou fut suffisamment grand, ils ont retourné le tambour pour en travailler l'autre extrémité. Là aussi, ils ont mis de la terre puis fait du feu. Avec un bambou sec allumé ils ont ensuite brûlé et creusé le "cou” de l'instrument jusqu'à percer un trou central de taille moyenne. Alors ils ont entièrement retiré l'écorce qui recouvrait l'extérieur de la pièce de bois. Ils ont coupé bien nettement la tête et le cul de l'instrument et ils ont à nouveau regardé ce que l'eau avait apporté; puis ils sont allés chercher une peau de serpent [comme membrane du tambour.]

Ils ont repéré un sindre' mena'a' [un python] dont ils ont retiré la peau après l'avoir tué. Ils en ont jeté les os, ne gardant que la peau et mangeant la chair. Ils ont coupé cette peau en plusieurs morceaux qu'ils ont tendus sur une armature de rotin. Tout le monde a fait ça. Ensuite ils les ont mis au soleil, afin de sécher le sang du serpent, pour que ces peaux ne pourrissent pas et durcissent. Observant encore ce que l'eau leur avait montré, ils virent une feuille de l'arbre so'o', dont ils ont déposé de la sève sur le pourtour de l'ouverture du tambour [là où sera collée la peau], et de la cire d'insecte unda' $a$ '. Certains partirent à la recherche d'un so'o', d'autres cherchaient 
des unda'a' dans des trous d'arbres. Ils ont préparé cette cire en mettant les insectes dans des longues feuilles de pebikwae (une herbe non identifiée) puis en les chauffant dans des entrenœuds de bambou. Ils l'ont ensuite fait refroidir. Ils ont alors frotté l'ouverture des tambours avec l'écorce de so'o'. Et, tous en même temps, ils ont installé les peaux tendues. Ils ont pris la cire d'insecte et ont cherché à accorder l'instrument [en collant plus ou moins de boulettes de cire sur la membrane en peau de serpent]. Ils ont essayé de battre les tambours et ceux-ci ont produit le bon son.

Ce sont des tambours du même type que nous-mêmes fabriquons et battons. Le sens profond de cette histoire, nous ne le connaissons pas. Nous avons seulement entendu nos anciens la raconter. C'est ainsi que nous chantons jusqu'à l'aube. Quand nous battons les tambours, c'est pour faire fuir le pisingen siwi du mort. On pense que l'esprit est encore parmi nous quand on enterre le mort et que l'on fabrique les aziara' [des parures de deuil].

Une autre version du mythe reprend et développe l'épisode de l'identification graduelle des matières premières et de la fabrication du premier tambour des humains.

Ils [les ombo'] l'avaient fait rêver. Ils lui avaient bien expliqué tout et il est arrivé directement au bon endroit. Il a coupé un bout d'écorce avec son herminette de pierre; il a senti son odeur et l'a comparée avec celle du morceau d'écorce qu'il avait [pris parmi les éléments attachés au tambour] : c'était la même odeur, alors il a su que c'était le bon arbre. Il l'a abattu et en a découpé un segment, vers le milieu du tronc. Il l'a porté sur l'épaule puis l'a laissé quelque part dans la forêt. Les ombo', on ne peut les voir, mais c'est d'eux que vient le songain. Le tambour apparu en pleine lumière, l'homme l'a pris. L'autre [resté sous l'eau], ce sont les ombo' qui le battent, cachés. Aujourd'hui comme hier, on ne peut voir les ombo'. Mon père a entendu cette histoire, et moi aussi. [Un autre informateur, qui tenait ce jour-là le rôle de traducteur du premier, m'explique alors que le héros avait cherché à attraper l'autre tambour en mettant la main dans l'eau, mais l'objet était parti sous l'eau. Puis le premier narrateur annonce qu'il va terminer l'histoire.]

Muni de son herminette, il est revenu là où il avait laissé le morceau de bois et il s'est mis à le creuser. Il l'a creusé jusqu'au milieu d'un côté, puis il l'a retourné. Son rêve lui avait bien expliqué comment procéder. Il a fait une encoche [pour repérer jusqu'où il avait perforé le cylindre de bois]. Il l'a retourné, et il a évidé l'autre extrémité. Là aussi, il s'est arrêté de creuser au milieu. Puis il est parti en forêt car il voulait un morceau de miye'. Avec son herminette, il a coupé un miye', et il l'a bien taillé en pointe. L'ayant affûté, il l'a emporté là où il s'était installé pour travailler et il a percé le "cou" au milieu du tambour avec cette pointe en miye"4. Il est ensuite allé se procurer un rotin épineux afin de s'en servir pour gratter l'intérieur du songain [en glissant cette lime végétale à travers le petit trou obtenu par percussion avec la pointe de miye']. A l'aide du rotin, il a déchiqueté l'intérieur mou du rondin. Ce rotin-là s'appelle sookwa' [une autre version parlait de nongwaye]. Pour la partie basse du tambour, il a d'abord dégagé un conduit au centre du morceau de bois jusqu'au "cou". On appelle cet endroit songain gu'no, comme le cou des hommes. Alors il a creusé la bouche du tambour, qu'on appelle songain mangain. Le bas du tambour, il l'a creusé tout doucement, en remontant vers le haut. Et il l'a appelé i'ke' mangain, "cul”. Il avait fait ce travail en restant caché, et quand il eut terminé l'instrument, il l'a apporté dans sa maison pour le faire sécher au-dessus du foyer. Puis il est reparti en forêt.

Il se baladait et capturait plein de petits serpents. Il leur retirait la peau, mais il ne le faisait pas correctement, alors il recommençait; et ça a continué comme ça longtemps. Il est retourné observer la peau de python [du tambour que lui avaient donné les ombo']. Il l'a regardée très attentivement : c'était une grande peau, alors il est reparti dans la forêt profonde pour en chercher une. Il ne l'a pas trouvée et il est revenu dormir chez lui. Ce même homme -le ombo' pardi !- lui a envoyé un nouveau rêve et lui a expliqué combien d'espèces de serpents ont une peau 
suffisamment grande [pour faire une membrane de tambour] : "Tu dois prendre la peau de trois serpents seulement: sobe', mena'a, awine"".5. Ayant reçu ce rêve, il a repris sa quête et a finalement rencontré un serpent, qu'il a tué. Il lui a retiré la peau et a pris un gros rotin dont il a fait un cercle. Ayant tendu la peau sur ce cercle, il en a gratté la chair qui y adhérait encore; il l'a bien nettoyée. Puis il a mis au soleil la peau tendue de cette façon. Quand le soleil l'eut fait sécher, la peau s'était agrandie [sous l'action des liens la tirant vers la périphérie du petit dispositif]. Une fois tout à fait sèche, il a essayé de l'installer sur le tambour en la mouillant avec de l'eau pour la ramollir. Il a alors cherché un arbre so'o' dont il a retiré l'écorce pour en dégager la sève poisseuse. Il a enduit l'ouverture du tambour avec cette sève, puis il l'a recouverte de la peau du serpent en entourant son rebord avec une cordelette [prise à sa femme] $]^{6}$ Ayant bien fixé la membrane, il a mis le tout à sécher au-dessus du feu, car la peau de serpent était détendue. Puis il a battu le tambour : ça ne rendait pas le bon son! Il a alors pensé à coller des crottes de son nez sur la peau du serpent et il a à nouveau battu le tambour : mais ça ne faisait pas le bon bruit! Il est retourné regarder l'instrument [d'origine], il l'a senti et il a bien noté son odeur. Il a cherché un arbre [dont l'espèce n'est pas précisée car il en existe des dizaines qui font ici l'affaire], jusqu'à en repérer un sur lequel il y avait plein de petits insectes. Il a coupé en haut et en bas l'écorce [sous laquelle ces animaux vivent] et il a retiré toutes ces unda'a [des alvéoles de cire]. Cette cire, il l'a mise dans un entrenœud de bambou qu'il a posé dans le feu. Après avoir chauffée la cire, il l'a fait refroidir et il a modelé des boulettes [pupa'] qu'il a fait adhérer sur la membrane en peau de serpent. Et il a essayé de battre le tambour : ça a explosé formidablement! À lui couper le foie! Tous les hommes, qui étaient chez eux, se sont rassemblés autour de lui; tous !

Au-delà de sa clarté et du niveau de détail qui le caractérise - au point qu'il ne déparerait pas parmi ces chaînes opératoires que les technologues décrivent et placent au centre de leurs recherches-, ce mythe technologique est remarquable par l'importance accordée au percement du "cou" du tambour. Ce dernier est l'un des rares objets qu'une partie seulement des hommes déclarent savoir façonner; mais, parmi tous les objets ankave, il est le seul dont la fabrication soit expliquée dans un récit mythique, alors qu'il en est d'au moins aussi complexes à produire. Il ne serait pas surprenant que la mémoire collective s'attarde sur les procédés particuliers que demande la reproduction, génération après génération, du cadeau fait par les ombo' aux humains. Quoi qu'il en soit, au sein des opérations techniques originales qu'il y a lieu d'accomplir, le percement du goulot d'étranglement qui met en relation les deux vasques des tambours mérite toute l'attention de ceux qui les fabriquent. Au point que l'on peut se demander (il ne s'agit ici que d'une hypothèse) si le façonnage du " cou " du tambour ne revêt pas pour les Ankave une importance qui dépasse sa difficulté technique objective. Mais les mythes insistent sur un autre aspect des tambours.

Membrane sonore ou seuil d'éternité ?

16 Après avoir résumé à sa manière la découverte de la mare aux tambours et les premières étapes de la fabrication de l'instrument, une autre variante du mythe aborde l'étonnante provenance de la membrane sonore. L'histoire résume d'abord l'habituelle découverte de l'instrument et le façonnage de sa première copie. Puis le héros en train de fabriquer le tambour s'adresse à son enfant :

Cet homme a dit à son enfant " Tu dois me couper la peau, celle du cou ". Et quand il n'a plus eu de peau sur le cou, il est devenu un serpent mena'a, qui est un gros python, et il est parti. Son enfant a installé la peau sur le tambour. Il l'a frappé : il rendait un très bon son. Auparavant, l'homme-serpent avait dit à son fils de prendre de la cire unda'a' car le jeune homme voulait y mettre des crottes de nez. Il 
avait dit à son enfant “ Tu vas retirer ma peau, mais tu ne dois pas me tuer : je ferai plein de petits que tu pourras occire pour faire des membranes de songain". Après avoir perdu sa peau, ce serpent est allé dormir dans un arbre u'pongwain, un grand arbre qui n'existe que plus bas [en direction de la plaine de Papouasie], plus bas que Peekwa, juste un peu plus bas.

Dans une autre version de ce mythe, le père généreux qui offre la peau de son cou précise que l'opération "ne [le] fera pas mourir" et son esprit indique en rêve au héros qu'il doit capturer des serpents, et comment s'y prendre. Mais, c'est en écoutant une troisième variante de ce sacrifice par lequel un homme offre sa peau à l'humanité ankave que je devais commencer de comprendre l'importance accordée à l'origine de la membrane des songain.

“Ayant perdu sa peau" pour le plus grand bénéfice d'un Ankave qui en couvrit le premier tambour, le serpent de cette histoire alla donc dormir dans un arbre u'pongwain, quelque part vers les basses terres :

Ce serpent mangeait tous les enfants des hommes, garçons et filles. Toujours il faisait ça. Un homme est monté [dans la forêt d'altitude]. Il voulait trouver des marsupiaux en brousse. Il vit son chien qui rapportait ce serpent [il s'agit de celui en lequel s'était transformé le père qui avait offert sa peau]. Il y avait là un petit enfant qui voulait tirer une flèche sur un lézard. Sa mère lui dit: "Tu dois te munir de ce couteau de bambou. Si le python vient te manger, ne lâche pas ce couteau". Le serpent avala l'enfant et, en même temps, le couteau. Une fois à l'intérieur, le garçon coupa le ventre du serpent et en sortit. Et l'enfant resta comme ça [sur place]. Sa mère alla le chercher là où disparaissaient les enfants, là où l'herbe était pliée par le serpent lorsqu'il les entourait. Elle y alla et vit le serpent avec du sang alentour, celui de l'enfant et celui de l'animal. Le garçon était là, les bras pendants, après avoir découpé le ventre du serpent. Ayant vu le python, la mère alla chercher beaucoup de gens. Elle le leur montra et dit: "C'est ce serpent qui a mangé tous ces enfants ". Elle rassembla plein de monde. Elle voulait tuer ce python. Ils avaient pris des herminettes de pierre, des masses d'armes kara et des arcs et des flèches. Et alors ils ont tiré dessus et l'ont tué pour de bon. Et ils sont allés chercher de la nourriture [à consommer en même temps que l'animal]. Le serpent était trop gros pour être rapporté au village. Ils ont rapporté des légumes à feuilles, des taros, des patates douces, des bananes. Puis ils ont coupé le python en morceaux qu'ils ont mis à cuire dans un four semi-enterré. [Le moment venu], ils ont ouvert le four et en ont retiré le serpent. Ils l'ont débité et distribué. Ce n'était pas bien cuit et ils ont mis ces morceaux dans leurs filets de portage en se disant qu'ils devraient s'assembler à nouveau pour le recuire. Ils ont cherché la tête et la queue du serpent sans les retrouver. Ils les ont [finalement] retrouvés, mais les deux bouts s'étaient recollés! Puis, les portions mises dans les filets se sont jointes d'elles-mêmes à la tête et à la queue !

Après s'être ainsi reformé, le serpent est resté à veiller. Il voulait occire ceux qui l'avaient tué. Tel le vent [tourbillonnant], il a encerclé toutes les maisons et tué tout le monde. Puis il est resté là à penser " Pourquoi ai-je tué tous ces gens ? " Il était là, redevenu un homme. À des hommes, il a dit: "Toute ma viande il l'ont mangée. Vous devez me la payer! " Ils lui ont montré des brasses de cauris, des décorations corporelles, de grands coquillages memia, mais il n'en voulait pas. Ils lui ont alors dit : "Si on te donne deux jeunes femmes, ça va ? - Oui ". Et les deux jeunes femmes ont épousé cet homme-serpent. Il est parti, suivi par les femmes. La nuit les a surpris sur la route, et ils ont dormi à un endroit couvert d'herbe. À l'aube, ils sont repartis. Les parents des deux femmes restaient là en tendant l'oreille. Ils leur avaient donné des herminettes en leur disant "La nuit venue, vous devrez couper du bois de chauffe avec ces outils". Arrivés à un palmier azo'o (Heterospathe sp.), ils ont grimpé le long de son tronc. L'homme d'abord, pendant que ses femmes restaient au sol. L'homme était redevenu un serpent pour monter à cet azo'o. Arrivé 
au sommet, il s'est retourné pour faire monter ses deux épouses. Les deux femmes étaient encore sur sol et elles lui ont attrapé la queue et elles l'ont coupée; chacune son tronçon. Les parents [des épouses du serpent] ont alors entendu "Kukukuku" [c'est-à-dire le bruit du tonnerre, akerukwa']. Ils se sont dits “ Il n'est pas mort ". Ce serpent s'appelle Omo. Les femmes étaient Makie' et Toe'. Elles se tournent le dos et sont devenues un rocher qui est près de Rabaul, à Siesi Island.

Outre son cannibalisme, le serpent géant présente la particularité -d'autant plus fondamentale qu'elle est partiellement liée à cette peau dont parle toute l'histoired'être immortel. Bien que non explicite dans le mythe, cette qualité de l'hommeserpent auto-sacrifié ne fait aucun doute. Comme tous les serpents, celui-là abandonne périodiquement sa peau sans mourir, ce qui en fait un bon candidat pour incarner l'immortalité. Mais, surtout, dans le mythe des enfants dévorés, le serpent se reconstitue et reprend vie alors qu'il a été estourbi, découpé en morceaux et à moitié cuit, ce qui constitue un indubitable gage de vigueur! L'immortalité du serpent est partagée par l'homme qui offre sa peau, soit parce qu'il prend soin d'annoncer à son fils que l'opération "ne [le] fera pas mourir", soit parce qu'il devient lui-même un serpent. Que la chaîne opératoire mythique insiste sur la nécessité de faire sécher la peau du serpent avant de l'installer sur le tambour "afin qu'elle ne pourrisse pas" confirme que la non-putréfaction, l'auto-régénération et, pour dire bref, l'immortalité ont quelque chose à voir avec l'insistance que met la culture ankave à charger de significations la membrane sonore de l'objet que les hommes reçurent des ombo'. Par un bruit de tonnerre, les tambours Ankave éloignent les pisingen siwi du séjour de ceux qui leur survivent. Mais ce raffut semble bien tenir quelque chose de ses mystérieuses fonctions des qualités d'une membrane inaltérable qui est elle-même l'élément conférant son immortalité à l'être dont elle provient.

De l'étude de ces mythes, du déroulement des rituels et de l'observation de l'ensemble tambour-porteur de masque nowimbere', il résulte que le tambour sablier joue un rôle capital dans l'expulsion des esprits des morts récents : attiré par les bras du masque nowimbere', le spectre pisingen siwi est envoyé vers l'au-delà par le tintamarre que produit la membrane du tambour. Ce faisant, l'esprit du mort franchit deux parties de l'instrument sur lesquelles les mythes d'origine des cérémonies songain sont particulièrement diserts : l'étranglement qui relie les deux chambres du tambour, et la membrane en peau de python, qui joue le rôle d'un seuil d'éternité.

De fait, dès leur origine, les tambours sont placés sous le signe de l'échange et du passage entre le monde des hommes et celui des monstres cannibales. Ils sont présents des deux côtés de la surface de l'eau, puisqu'un autre tambour, celui des ombo', est reparti dans les profondeurs de la mare. Au moment où il passa d'un tourbillon chantant aux mains d'un ancêtre ankave, l'instrument conservé par les humains se trouvait de surcroît exactement dans la position qui désigne la fonction que lui assignent les ombo' et, à travers eux, la culture ankave : celle d'un tube reliant les deux faces d'une même entité, la société ankave avec ses vivants et ses morts. Dès lors, les tambours-sabliers nous apparaissent pour ce qu'ils sont d'abord: des entonnoirs psychopompes, c'est-à-dire l'étroit canal que doit emprunter un spectre pour quitter le monde des vivants et se rendre dans celui des ombo'.

Mythe, rite et technique

Au-delà de la place peu courante que des chaînes opératoires tiennent dans ces mythes, c'est la manière dont ils mêlent d'une façon inextricable l'histoire de l'origine des tambours, les rituels auxquels ces derniers donnent leur nom et leur mode de 
fabrication qui doit retenir notre attention. Ces récits qui expliquent tout autant l'origine des rites songain que celle de la transformation de billes de bois en instruments sonores parlent à l'unisson d'une procédure technique répétée génération après génération (chaque fois qu'un tambour est mis en forme) et de l'action magique (pour nous) qui fait passer le fantôme d'un mort récent de ce monde vers celui des ombo'. Dans cette affaire, il serait aussi injustifié que malencontreux de distinguer des références à des actions matérielles tangibles, comme l'emploi de la liane nongwaye "qui a des épines" ou les étapes du perçage du "cou" des tambours, de l'invisible fonction psychopompe des instruments : c'est précisément la confusion dans un même récit d'actions réelles et d'autres, pour nous imaginaires, qui donne au mythe les sens que les Ankave lui attribuent explicitement, à savoir l'explication, la justification et l'origine des rituels qui les débarrassent de leurs morts, en même temps qu'ils donnent l'oubli aux vivants.

Mythe, technique et rituel sont tout aussi enchevêtrés lors du piégeage des anguilles dont la chair fumée est offerte à certains cousins croisés (réels ou classificatoires) à l'extrême fin des cérémonies de lever de deuil au cours desquelles on frappe des tambours (Lemonnier 1993a). Constituées d'un cylindre d'écorce dont une extrémité est obturée par des barreaux tandis que l'autre est munie d'une porte que la détente d'un arc referme violemment sur l'animal, les trappes utilisées présentent des caractéristiques mécaniques qui leur donnent une solidité bien supérieure à ce qui serait nécessaire pour résister à la vigueur des poissons capturés ${ }^{8}$. L'observateur ne peut manquer d'être frappé par les efforts et le soin déployés par les Ankave tout au long du processus de fabrication du piège pour accumuler l'énergie passive que le piège opposera aux débattements de l'animal capturé. Ces précautions redoublées apparaissent d'autant plus superfétatoires que l'on connaît en Nouvelle-Guinée d'autres pièges du même type qui ne présentent pas un tel luxe de renfort. Il ne fait aucun doute que la nasse résisterait aussi bien aux mouvements de l'anguille si ses ligatures étaient moins élaborées, et tant la fabrication que l'installation de ces frettes s'accommoderaient de moins de force et de précision, voire de recherche esthétique. D'autre part, comparée à la parfaite solidité du dispositif, l'attention apportée à son ancrage au bord des torrents laisse à désirer : la robustesse du piège apparaît hors de proportion avec le risque de le voir emporté par les eaux. Pour les Ankave, la capture de l'animal semble importer moins que le maintien de son enfermement et que le contrôle de sa force.

Rapprochant cette constatation du mythe d'origine des anguilles - qui indique que les premiers de ces poissons étaient en fait issus du pénis sectionné d'un ancêtre qui avait la mauvaise habitude de copuler par surprise et à distance avec les femmes, jusqu'à ce que l'une d'elles coupe son membre importun-, j'ai avancé l'hypothèse qu'à la vigueur supposée des anguilles correspond une représentation des techniques de construction du piège, et du piège lui-même, qui met en avant l'accumulation d'une énorme énergie passive, capable de s'opposer avec succès à ce symbole de vitalité qu'est l'animal. Bref, tout se passe comme si l'objet lui-même et les onomatopées essoufflées de ses constructeurs garantissaient le statut symbolique de l'anguille. Ici, c'est l'analyste qui met le mythe en rapport avec ses trouvailles de technologue, mais les gestes de celui qui réalise un piège et l'imaginaire qui donne vie aux anguilles se renforçant mutuellement, une même confusion des domaines "technique" et “mythique" est à l'œuvre. Lors de l'une des étapes du processus de fabrication des 
trappes, ce mélange entre les deux ordres de phénomènes que nous isolons le temps de l'enquête est tel qu'il est impossible de distinguer l'action sur la matière (en l'occurrence, l'installation et l'essai de l'arc et du dispositif qui le maintient bandé jusqu'à ce qu'une anguille déclenche la fermeture de la nasse) du rituel qui rejoue le mythe.

Fait d'une branche élastique de1,5 cm de diamètre, cet arc est simplement fiché "à force " à l'arrière du cylindre et à la jonction des deux branches du "diapason " qui constitue l'armature principale du piège et relié à sa détente. Peu avant leur mise à l'eau par l'artisan (qui est aussi l'organisateur de la cérémonie de lever de deuil en vue de laquelle on va piéger des anguilles), toutes les trappes qui viennent d'être fabriquées sont ainsi alignées verticalement le long d'une frêle barrière érigée pour l'occasion, et les arcs formant ressort sont bandés. L'une des épouses de cet homme déclenche alors très rapidement les pièges les uns après les autres en touchant leur détente avec un bâtonnet en bois d'ingwi'i (Litsea sp.) à l'aide duquel elle vient de se mettre du sel végétal dans la bouche. Seule une femme mûre est réputée savoir correctement effectuer ce rituel, qu'elle apprend de son mari. L'odeur du sel, pense-t-on, attirera les anguilles, et il est précisé que sans cette pratique magique, “on n'attraperait qu'une anguille en un mois!" Mais on voit aussi qu'une femme effectue ici un geste semblable à celui par lequel la femme mythique met volontairement en action le piège qui sectionne le pénis du fâcheux amant et crée du même coup l'ancêtre des anguilles. À ce moment, les piégeurs prononcent silencieusement une formule magique dans laquelle le bâtonnet tenu par la femme est décrit comme déclenchant l'arc qu'est le ressort du piège, puis il frotte les câbles de rotin des pièges à l'aide d'une noix magique (une graine de Mucuna albertisii) tout en murmurant quelques paroles. On achève alors d'orner les pièges avec diverses feuilles, strictement décoratives ou odoriférantes, dans le but d'attirer les futures proies.

Comme on le voit, la chaîne opératoire de la préparation des pièges et le rite qui assure leur efficacité ne font qu'un. Il serait par exemple impossible de décider si le claquement de la porte qui se referme à vide est un essai du dispositif ou une mise en scène rituelle de l'acte féminin qui donna aux pénis des hommes une taille adaptée à la sexualité. Deux conclusions s'imposent alors.

Ces exemples ankave soulignent d'abord, une fois de plus, à quel point il est illusoire de distinguer a priori les techniques des autres productions socioculturelles. Cinquante années de technologie culturelle ont amplement démontré que, du fait de l'inscription des représentations et des actions techniques dans toutes sortes de systèmes de pensée et de pratiques, nous (et les autres) mettons sans cesse en œuvre des techniques plus ou moins absurdes, non physiquement pertinentes, voire mal adaptées à leur fonction matérielle (Lemonnier 1993b). Comme tant d'autres y ont déjà insisté, chacun à sa manière, les tenants et aboutissants d'une activité technique dépassent toujours sa seule efficacité matérielle (par exemple, et presque au hasard: Cresswell 1996: 6; Gosselain 1999; Latour 1993; Lemonnier 1993b; Mahias 1993, 2002; Virolle-Souibès 1989). De la manière dont les Ankave mêlent mythes, rites et techniques, il résulte en particulier que distinguer les actes "rituels" par leur manque d'efficacité matérielle intrinsèque n'est pas satisfaisant.

Or, parmi les travaux récents sur le rituel, qui se concentrent désormais sur la compréhension des formes d'action qui seraient propres à ce type de productions socioculturelles, plutôt que sur leurs fonctions ou significations (Houseman \& Severi 
1994; Humphreys \& Laidlaw 1994), certains soulignent l'absence de “technical motivations " qui les caractériserait. Pourtant, si comme l'écrit Whitehouse (2002: 46) en accord avec Humphreys \& Laidlaw (1994 : 158), “what enables us to recognize an action as ritualized is a lack of "technical motivation" [...] with regard to at least some details of the prescribed action scheme", alors toute action technique comporte une dimension rituelle... L'heure est ici à rapprocher les termes " mythe " et " technique ", et non à confronter les multiples tentatives de définition du rituel avec nos trouvailles de technologues, mais notons que si les rituels sont incontestablement des "potentially puzzling forms of action" (Whitehouse 2002:46), cela ne suffit pas à les distinguer des techniques. Chacun, au fond de lui, sait bien que planter un clou n'est pas tout à fait la même chose que déposer un bâton d'encens en murmurant une formule, mais l'étude monographique des tambours et des pièges ankave montre que des portions entières de chaînes opératoires se confondent avec des actions rituelles. Au passage, gageons qu'approfondir ce rapprochement entre "technique" et " rituel " révèlerait rapidement un relatif manque de détail des descriptions de rituels, ce qui laisse de beaux jours au recueil de chaînes opératoires ${ }^{9} . .$.

30 La technologie culturelle n'est, assurément, qu'un point de vue (Bonte 1999: 15), celui qui, parmi une multitude de représentations et de pratiques socioculturelles, prend aussi en compte celles qui se traduisent par des actions sur la matière susceptibles de varier d'un peuple à l'autre. Et ce point de vue n'est pas destiné à construire seulement un savoir sur la "technique", mais un savoir sur les aspects les plus divers d'une culture ou d'une organisation sociale. L'imbrication des mythes, des rites et des pratiques techniques autour des tambours et pièges à anguilles ankave rappelle donc également qu'analyser les chaînes opératoires mises en œuvre lors de leur production ne devient pas seulement un moyen d'étendre une enquête à tous les registres documentaires d'une monographie à tiroirs : outre cette rassurante constatation que les ethnologues ne sont pas les seuls à penser et dire des actes techniques sous forme de séquences d'actions sur la matière, se pencher sur les dimensions les plus matérielles des objets, comprendre leur fabrication ou leur fonctionnement permet surtout de mettre au jour des pans entiers de systèmes de pensée ou de logiques sociales qu'on ne saurait repérer et comprendre autrement.

31 Comme pour toute recherche sur les rapports entre les techniques et d'autres dimensions de la vie sociale et des production culturelles, les analyses détaillées sont ici indispensables et disent nécessairement autre chose et davantage que ce que peut produire une caractérisation générale des phéno-mènes en jeu. Un peu comme lors de ces études sur la sacro-sainte " articulation" des forces productives et des rapports de production qui se contentaient de survoler l'action technique tout en ouvrant des voies fécondes, ne serait-ce qu'en explorant des impasses (Lemonnier 1980:4-7), on peut assurément dire beaucoup des rapports entre rites, mythes et techniques par une qualification abstraite des phénomènes en jeu (Bonte 1985 : 22-25, 1999). Mais, du fait de la nature même de ces phénomènes et de l'angle d'attaque qui définit la technologie culturelle, aller au charbon (ou au cambouis) et observer au plus près de l'action sur la matière est un moyen privilégié de cerner au mieux l'entrelacs de gestes et de paroles qui les caractérise.

Chez les Ankave, la façon de fabriquer des engins sonores ou des pièges fournisseurs de graisse et de protéines parle du deuil et de l'oubli des morts, de l'imputation du 
malheur et de la circulation de la vie entre les générations. Encore faut-il se donner la peine et les moyens de le montrer.

\section{BIBLIOGRAPHIE}

Balfet, Hélène

1975.“ Technologie ”, pp. 44-79, in R. Cresswell (ed.), Éléments d'ethnologie, vol. 2. Paris : Armand

Colin.

Balfet, Hélène (ed.)

1991.Observer l'action technique. Des chaînes opératoires, pour quoi faire ? Paris : Éditions du

CNRS.

Bonte, Pierre

1985." Faits techniques et valeurs sociales : quelques directions de recherche ", Techniques \&

culture $5: 19-48$.

1999.“ Travail, techniques et valeur : Contribution (nouvelles) au débat sur rites et techniques ", in J.-L. Jamard, A. Montigny, F.-R. Picon (eds), Dans le sillage des techniques. Hommage à Robert Cresswell. Paris : L'Harmattan.

Byrne, R.

1995.The Thinking Ape. Oxford : Oxford University Press.

Cresswell, Robert

1972." Les trois sources d'une technologie nouvelle ", pp. 21-27, in J. M. C. Thomas et L. Bernot (eds), Langues et techniques, nature et société, t. 2, Approche ethnologique, approche naturaliste. Paris : Klincksieck.

1996.Prométhée ou Pandore? Propos de technologie culturelle. Paris : Kimé.

Debary, O.\& A. Tellier

2004.“ Objets de peu : Les marchés à réderies dans la Somme ”, L’Homme 170 : 117-138.

Digard, Jean-Pierre

1979.“ La technologie en anthropologie : fin de parcours ou nouveau souffle ? ", L'Homme 19 (1) : 73-104.

Faure-Rouesnel, L.

2001.“ French anthropology and material culture ", Journal of Material Culture 6 (2) : 237-247.

Geistdoerfer, Aliette

1973.“ Leroi-Gourhan : méthode d'analyse des techniques ”, La Pensée 171 : 60-74.

Gille, Bertrand

1978. Histoire des techniques. Paris : Gallimard.

Gosselain, O. P.

1999." In pots we trust : the processing of clay and symbols in Sub-Saharan Africa ", Journal of Material Culture 4 (2) : 205-230. 
Houseman, Michael \& Carlo Severi

1994.Naven ou le donner à voir. Essai d'interprétation de l'action rituelle. Paris : Éditions du CNRS/

Éditions de la Maison des sciences de l'homme.

Humphrey, Caroline \& James Laidlaw

1994.The Archetypal Actions of Ritual : A Theory of Ritual Illustrated by the Jain Rite of Worship.

Oxford : Clarendon Press.

Joulian, Frédéric

1996." Comparing chimpanzee and early hominid techniques : some contribution to cultural and cognitive question ", pp. 173-189, in P. Mellars et K. Gibson (eds), Modelling the Human Mind. Cambridge : MacDonald Institute Monographs.

Julien, Marie-Pierre, Julie Poirée, Céline Rosselin, Mélanie Roustan, Jean-Pierre Warnier 2002." Chantier ouvert au public", Techniques \& culture 40 : 185-192.

Kratz, C. A

1994.Affecting Performance : Meaning, Movement, and Experience in Okiek Women's Initiation. Washington/London : Smithsonian Institution Press.

Küchler, S.

1988." Malangan : objects, sacrifice and the production of memory ", American Ethnologist 15 (4) : 625-637.

1997." Sacrificial economy and its objects : rethinking colonial collecting in Oceania ", Journal of Material Culture 2 (1) : 39-60.

Landzelius, K. M.

2001." Charged artifacts and the detonation of liminality ", Journal of Material Culture 6 (3) :

323-344.

Latour, Bruno

1993.La Clef de Berlin et autres leçons d'un amateur de sciences. Paris : La Découverte.

Leach, Jerry W.

2002." Drum and voice : aesthetics and social process on the Rai Coast of Papua New Guinea ", Journal of the Royal Anthropological Institute (N.S.) 8 : 713-734.

Lemonnier, Pierre

1976.“ La description des chaînes opératoires : contribution à l'analyse des systèmes techniques ", Techniques et culture (Bulletin) $1: 100-151$.

1980.Les salines de l'Ouest : logique technique, logique sociale. Paris/Lille : Éditions de la Maison des sciences de l'homme/Presses universitaires de Lille.

1983.“ L'étude des systèmes techniques, une urgence en technologie culturelle ", Techniques \& culture $1: 11-34$.

1993a." L'anguille chez les Ankave-Anga : matérialité et symbolique du piégeage ", pp. 1013-1026, in C.-M. Hladik et al. (eds), L'Alimentation en forêt tropicale : interactions bioculturelles et applications au développement. Paris : UNESCO.

1993b." Introduction ", pp. 1-35, in P. Lemonnier (ed.), Technological Choices : Transformation in Material Culture since the Neolithic. London : Routledge. 
2004.“ Savez-vous casser les noix, à la mode, à la mode... À quoi bon singer la culture ? ", in F. Joulian (ed.), Culture et significations chez les Anthropoïdes, les hommes préhistoriques et les humains actuels. Paris : Balland.

s/presse." Comment façonner un entonnoir psychopompe ? Une chaîne opératoire mythique en Nouvelle-Guinée ”, in F. Cousin (ed.), Hommage à Hélène Balfet (titre provisoire).

Leroi-Gourhan, André

1964.Le Geste et la parole. 1. Techniques et langage. Paris : Albin Michel.

Mahias, Marie-Claude

1993." Pottery techniques in India. Technical variants and social choice”, pp. 157-180, in P. Lemonnier (ed.), Technological Choices : Transformation in Material Culture since the Neolithic. London : Routledge.

2002.Le Barattage du monde. Essais d'anthropologie des techniques en Inde. Paris : Éditions de la Maison des sciences de l'homme.

Mauss, Marcel

1968." Les techniques du corps ”, pp. 365-383, in Sociologie et anthropologie. Paris : Presses Universitaires de France (1ère édition 1936, Journal de Psychologie 23/3-4).

Miller, D.

2000." The fame of Trinitis : websites as traps ", Journal of Material Culture 5 (1) : 5-23.

Virolle-Souibès, Marie

1989." Pétrir la pâte, malaxer du sens : Exemples kabyles ", Techniques \& culture 13 : 73-101.

Warnier, Jean-Pierre

1999.Construire la culture matérielle. L'homme qui pensait avec ses doigts. Paris : Presses Universitaires de France.

Whitehouse, Harvey

2002." Conjectures, Refutations, and Verification : towards a testable theory of "modes of religiosity" ", Journal of Ritual Studies 16 (2) : 44-59.

Whiten, A., Jane Goodall, W. McGrew, T. Nishida, V. Reynolds, Y. Sugiyama, C.E.G Tutin, R. W. Wrangham, C. Boesch

2000." Culture in Chimpanzees ", Nature 399 : 682-685.

2001.“ Chrating cultural variations in Chimpanzees ”, Behaviour 138 : 1481-1516.

\section{NOTES}

1. Lors d'un colloque au Max Planck Institut de Leipzig, j'ai émis l'hypothèse qu'un bon moyen d'approfondir notre connaissance des dispositifs mentaux qui sous-tendent les extraordinaires nuances " culturelles " observées chez les primates consisterait à raffiner l'observation et l'analyse de leurs gestes, outillés ou non; c'est-à-dire à recueillir des chaînes opératoires du types de celle qu'un ethnologue tente d'analyser. Il m'a été répondu que c'était " beaucoup de travail et très compliqué ". Assurément !

2. Avec le recul -entendre : compte tenu des découvertes cumulées de la technologie culturelle, de la sociologie de l'innovation et de la primatologie-, l'idée même que "les rapports des hommes entre eux " puissent être indépendants des objets qui les entourent fait désormais franchement sourire... (Lemonnier 1980 : 1-13). 
3. Je fais allusion aux remarquables articles que Miller (2000) et Landzelius (2001) ont respectivement consacrés aux sites Internet considérés comme des pièges et à la “ diplomatie du nounours " à laquelle recourent les mères (et, espère-t-on, les pères) pour pénétrer dans la bulle de la couveuse où leur bébé cherche la vie.

4. Outre le miye' (Garcinia sp.), qui comporte deux sous-espèces reconnues par les Ankave, on peut aussi utiliser pour cette tâche un autre arbre au bois particulièrement dur appelé itsuwe'wo (Fagraea sp.) qui présente l'intérêt de résister aux attaques des insectes.

5. Les Ankave utilisent en fait la peau de quatre types de " python " sur les sept qu'ils distinguent.

6. Trois arbres (komare, Duckera taitensis; kwia', Garcinia sp. et sirige Evodia sp.)

fournissent pour ce collage de la peau de python sur l'ouverture du tambour une sève qui demande à être chauffée préalablement; on utilise aussi le jus d'une igname sauvage, tomwe' undaa', qui est comestible.

7. Onomatopée qui, jusqu'à plus ample informé, n'a aucun rapport avec le terme par lequel les Anga étaient désignés par leurs voisins. D’autres versions de ce même mythe du serpent cannibale précisent que l'on entendit alors le bruit de la pluie et le tonnerre. Le " bruit de pluie " fait par le serpent est alors "klokloklo".

8. L'écorce et ses renforts se présentent comme une structure dont chaque élément est plusieurs fois lié à l'ensemble dont il participe et garantit la robustesse. Ainsi, non seulement toutes les séries de spires jointives jouant le rôle de frettes sont étroitement plaquées à l'écorce, mais elles sont aussi maintenues latéralement en place en étant fortement “ ancrées " le long de l'exo-squelette constitué par une longue pièce de bois en forme de diapason. Plusieurs de ces frettes superposées sont de surcroît solidarisées par une ligature traversant l'écorce. Comme si tout cela ne suffisait pas encore, des tendeurs en forme de " 8 " resserrent sur elles-mêmes les spirales extérieures des deux extrémités en même temps qu'elles les plaquent sur l'écorce (Lemonnier 1993a :

1023-1024).

9. Il ne manque évidemment pas de remarquables exceptions, par exemple Kratz (1994).

\section{RÉSUMÉS}

Pour expliquer l'origine des tambours sabliers qu'ils frappent nuit après nuit afin de chasser les esprits des morts récents, les Ankave-Anga racontent une série de mythes dans lesquels le mode de fabrication de ces objets est exposé avec un soin digne d'une chaîne opératoire de «technologue ». Outre la question générale des frontières entre techniques, mythes et rites -ici impossibles à tracer-, cette étude de cas démontre que, sans une fine connaissance de la production matérielle de ces objets, il serait vain d'espérer comprendre les cérémonies de secondes funérailles dans cette partie de la Papouasie Nouvelle-Guinée.

Mythical operational sequences

In order to explain the origin of the hour-glass drum they beat for nights on end to chase away the spirits of the recent dead, the Ankave-Anga tell a series of myths that detail the making of 
these artifacts befitting a "technologist's » operational sequence (chaîne operatoire). In addition to the general question of defining the limits of techniques, myths, and rituals - which are impossible to delineate in the present case-, this study demonstrates that a fine-grained knowledge of the material production of these drums is an absolute prerequisite to understand secondary funeral rituals in this part of Papua New Guinea

Míticas cadenas operatorias

Para explicar el origen de los tambores ampolletas que se tocan noche tras noche para auyentar los espíritus de los muertos recientes, los Ankave-Anga cuentan una serie de mitos que describen el modo de fabricación de estos objetos con una precisión comparable a la de un "tecnólogo ». Además de la cuestión general de las fronteras entre técnicas, mitos y ritos - que no se pueden trazar en el caso presente- este estudio de caso demuestra que sin un conocimiento completo de la producción material de estos objetos, no se puede lograr la comprensión de las ceremonias de segundo entierro en esta parte de Papuasia Nueva Guinea.

\section{INDEX}

Mots-clés : chaîne opératoire, Papouasie Nouvelle-Guinée, Anga, mythe, rituel, deuil

Keywords : Papua New Guinea, myth, operational sequences, ritual, mourning

\section{AUTEUR}

\section{PIERRE LEMONNIER}

Centre de recherche et de documentation sur l'Océanie, CNRS, EHESS, Université de Provence, Marseille 